\title{
Correction to: The association between metabolic risk factors, nonalcoholic fatty liver disease, and the incidence of liver cancer: a nationwide population-based cohort study
}

\author{
Yu-Guang Chen ${ }^{1,2} \cdot$ Chih-Wei Yang $^{3} \cdot$ Chi-Hsiang Chung ${ }^{4,5} \cdot$ Ching-Liang Ho $^{1} \cdot$ Wei-Liang Chen $^{6} \cdot$ Wu-Chien Chien ${ }^{4,5}$
}

Received: 2 February 2022 / Accepted: 2 February 2022 / Published online: 16 February 2022

(c) Asian Pacific Association for the Study of the Liver 2022

\section{Correction to: Hepatology International https://doi.org/10.1007/s12072-021-10281-9}

The above article was published with few incorrected sentences we did not find before.

The changes in the text

1. In the conclusion part of the abstract, we state "Moreover, coexisting multiple metabolic risk factors would dampen the risk of developing $\mathrm{HCC}$ in patients with NAFLD." In fact, this should state "Moreover, coexisting multiple metabolic risk factors would increase the risk of developing HCC in patients with NAFLD."

The original article can be found online at https://doi.org/10.1007/ s12072-021-10281-9.

Wei-Liang Chen

weiliang0508@gmail.com

Wu-Chien Chien

chienwu@ndmctsgh.edu.tw

1 Division of Hematology/Oncology, Department of Internal Medicine, Tri-Service General Hospital, National Defense Medical Center, Taipei, Taiwan

2 Cancer Institute, University College London, London, UK

3 Division of Gastroenterology, Department of Internal Medicine, Tri-Service General Hospital, National Defense Medical Center, Taipei, Taiwan, ROC

4 School of Public Health, National Defense Medical Center, Taipei, Taiwan, ROC

5 Department of Medical Research, Tri-Service General Hospital, National Defense Medical Center, Taipei, Taiwan, ROC

6 Department of Family Medicine, Tri-Service General Hospital, National Defense Medical Center, No. 325, Section 2, Cheng-Kung Road, Neihu District, Taipei City 11490, Taiwan, Republic of China
2. In the second paragraph of the result, we state "This result emphasized that coexisting MetS in NAFLD patients dampens the risk of developing HCC." In fact, this should state "This result emphasized that coexisting MetS in NAFLD patients increased the risk of developing HCC."

3. In the first paragraph of the discussion, we state" Coexisting NAFLD in patients with multiple metabolic risk factors (Metabolic syndrome status) would dampen the risk of HCC compared to that in the MetS alone population." In fact, this should state "Coexisting NAFLD in patients with multiple metabolic risk factors (Metabolic syndrome status) would increase the risk of HCC compared to that in the MetS alone population.

4. In the third paragraph of the discussion, we state "while metabolic risk factors serve as aggravated factors for dampening this process." In fact, this should state "while metabolic risk factors serve as aggravated factors for this process."

Publisher's Note Springer Nature remains neutral with regard to jurisdictional claims in published maps and institutional affiliations. 